\title{
Adverse events associated with acupuncture: three multicentre randomized controlled trials of 1968 cases in China
}

\author{
Ling Zhao', Fu-wen Zhang ${ }^{2}$, Ying Li $i^{1}, X_{i}$ Wu$^{1}$, Hui Zheng ${ }^{1}$, Lin-hao Cheng ${ }^{3}$ and Fan-rong Liang ${ }^{* *}$
}

\begin{abstract}
Background: In order to evaluate the safety of acupuncture in China objectively, we investigated the adverse events associated with acupuncture based on three multicentre randomized controlled trials (RCTs) to assess the safety of acupuncture, identifying the common types of acupuncture adverse events, and analysing the related risk factors for their occurrence.

Methods: This observational study included patients who received acupuncture from three multicentre RCTs respectively for migraine, functional dyspepsia and Bell's palsy. The 1968 patients and their acupuncturists documented adverse events associated with acupuncture after treatment. We collected data about adverse events due to acupuncture treatment from their case report forms. We analysed the incidence and details of the adverse effects, and studied the risk factors for acupuncture adverse events with non-conditional logistic regression analysis.

Results: Among the 1968 patients, 74 patients (3.76\%) suffered at least one adverse event throughout the treatment period. We did not observe the occurrence of serious adverse events. 73 patients with adverse events recovered within 2 weeks through effective treatment such as physiotherapy or self-treatment. A total of 3 patients withdrew because of adverse events. There were 9 types of adverse events related to acupuncture, including subcutaneous haematoma, bleeding, skin bruising and needle site pain. Subcutaneous haematoma and haemorrhage in the needling points were the most common adverse events. Age and gender were related to the occurrence of acupuncture adverse events. The older the patients were, the higher the risk of adverse events was. In addition, male patients had slightly higher risk of an adverse event than female patients.
\end{abstract}

Conclusions: Acupuncture is a safe therapy with low risk of adverse events in clinical practice. The risk factors for adverse events (AEs) were related to the patients' gender and age and the local anatomical structure of the acupoints. AEs could be reduced and mitigated by improving the medical environment, ensuring a high technical level of the acupuncture practitioners and establishing a good relationship of mutual trust between doctor and patient.

Trial Registration: ClinicalTrials.gov: NCT00599586, NCT00599677, NCT00608660

\section{Background}

Acupuncture has been practiced in China for thousands of years as part of the Traditional Chinese Medicine (TCM). More recently, it has gradually won acceptance in western countries as an alternative or complementary treatment for various conditions, including chronic pain

\footnotetext{
* Correspondence: acuresearch@126.com

'College of Acupuncture and Massage, Chengdu University of TCM,

Chengdu, Sichuan 610075, China

Full list of author information is available at the end of the article
}

syndrome [1-5]. Because of its widespread use, the safety of acupuncture is of increasing importance. Most sideeffects encountered in acupuncture practice are mild, but life-threatening adverse events such as organ injury have been reported [6]. In the past few years, AEs associated with acupuncture treatment in foreign countries have been published in the form of systematic reviews or surveys [7-10], but acupuncture-related AEs that occurred in China have not received attention as many Chinese practicing acupuncturists do not regularly

\section{Biomed Central}


monitor and report AEs. In this paper we report AEs due to acupuncture in 1968 cases of patients and analyse the risk factors for their occurrence, based on three multicentre RCTs of acupuncture, to provide an objective evaluation of acupuncture safety in China. This study is expected to be useful for acupuncture practitioners as well as educators and health care decisionmaking departments.

\section{Methods}

\section{Patients}

1968 outpatients came from three multicentre RCTs in China. These three RCTs are "An RCT to treat migraine with acupuncture" (MI-RCT), "An RCT to treat functional dyspepsia with acupuncture" (FD-RCT), and "An RCT of acupuncture and moxibustion to treat Bell's palsy according to different stages" (BP-RCT).

Three RCTs were registered respectively (NCT00599586, NCT00599677, and NCT00608660) at the U.S. clinical trial registry (http://clinicaltrials.gov/). According to the criteria of the International Classification of Headache Society, patients who met the diagnosis of migraine with or without aura were included in the MI-RCT study. In the FD-RCT study, patients diagnosed with meal-induced dyspeptic symptoms and epigastric pain according to the Rome III Diagnostic Criteria for functional Gastrointestinal Disorders. Patients meeting the diagnosis of Bell's palsy according to Chinese Medicine and Western Medicine were enrolled in the BP-RCT. The three RCTs were completed in 16 hospitals located in five provinces (Sichuan Province, Hunan Province, Hubei Province, Tianjin and Shandong Province). 1968 cases of patients were monitored from December 2007 to October 2009.

\section{Design}

Three RCTs were performed in accordance with the principles of the Declaration of Helsinki (Edinburgh 2000 version), and trial protocols were approved by the Affiliated Hospital of Chengdu University of Traditional Chinese Medicine review board and ethics committee. The National Clinical Trial Centre of Chinese Medicine, Chengdu, Good Clinical Practice (GCP) Centre in China was responsible for the central randomisation and data management. The inclusion criteria and protocols of the three RCTs have been described in detail elsewhere [11-13].

$\mathrm{AE}$ is defined as an unfavourable medical event that occurs during or after the treatment regardless of causal relationship [10]. Serious adverse effects (SAEs) refers to those that caused hospitalisation, extended duration of hospitalisation, disability, impaired ability to work, death or were life threatening, resulting in events such as congenital malformations in the process of the clinical trials. AE and SAE were defined a priori from the literature and the State Food and Drug Administration
(SFDA) in China. AEs include subcutaneous haematoma, minor haemorrhage, serious pain, fainting and local infection, and SAEs include spinal cord injury, punctured organs, convulsions and pneumothorax. All the definitions were expounded in the clinical work manual of each trial.

During the clinical trial, physicians and patients were asked to evaluate AEs/SAEs associated with acupuncture and were recorded in the case report form (CRF). After each acupuncture treatment cycle, each patient was asked to complete a questionnaire (see additional file 1: Adverse Events Questionnaire for Patients) about whether they suffered excessive pain in the needle points, nausea, dizziness, aggravation of illnesses or other discomforts during the treatment, and were evaluated if there was bleeding, haematoma, bruising, acupuncture fainting, lag needle, broken needle, forgotten needle, etc. If any of the above adverse reactions happened, doctors should record them in the AEs reports (see additional file 2: Adverse Events Reports for Acupuncturist) including the type of $\mathrm{AE}$, when it occurred, how long it lasted, the severity, location, any remedial actions and when they were alleviated, the relevance to acupuncture therapy and whether the patients quitted the trial. Monitors, appointed by the trial organisers, directly tracked AEs and periodically verified the integrity and authenticity of records for quality control [14].

\section{Interventions}

Patients in the three RCTs were blind selected to which acupuncture treatment they received. All enrolled patients received a 4-week acupuncture treatment and a 3 -month follow-up period. After randomisation, patients received 20 treatments over a period of 4 weeks, and the treatments would be administered once per day for 5 continuous days, with a 2 day rest interval. The treatment courses of the three RCTs were designed according to the acupuncture protocol used in Chinese clinical acupuncture practice. All acupuncture practitioners had undergone either at least 8 years of acupuncture training and were qualified TCM doctors or associate chief TCM doctors with more than 10 years of clinical experiences. Acupoints and manipulation procedures in MI-RCT and FD-RCT were standardised, and BP-RCT was performed using semi-standardised acupoints and standardised manipulation. Details of acupoints selected and manipulation in each trial were shown in additional file 3 (Details of acupoints selected and manipulation in each trial) and acupoints locations was shown in Figure 1. Sterile disposable one-time-use needles (Hwato Needles, Sino-foreign Joint Venture Suzhou Hua Tuo Medical Instruments Co., China) were used to achieve "de Qi" sensations. Patients in MI-RCT, FD-RCT and group 3 of $\mathrm{BP}-\mathrm{RCT}$ received electro-stimulation and the other patients were stimulated manually. 


\section{Acupoints Locations}
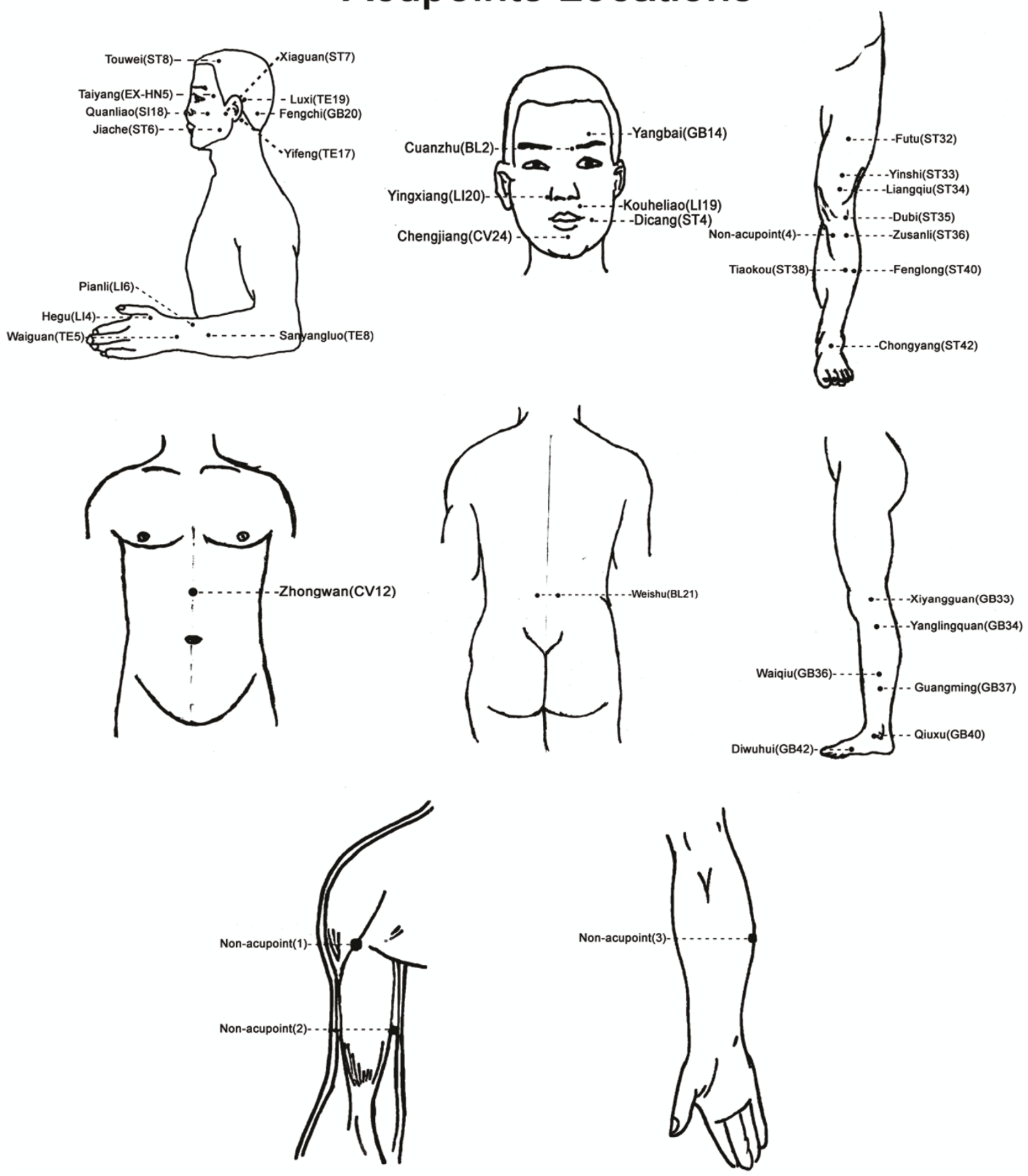

Figure 1 Acupoints Locations. Locations of acupoints used in the three randomized controlled trials.

\section{Data monitoring and Statistics}

According to standard operating procedures (SOPs) for clinical research data management, clinical trial data entry and management were commissioned by the GCP centre of Chengdu using Remote Clinical
Data Management Systems (RCDMS). All the results were entered twice and then checked. For inconsistent values we checked the CRFs item by item in order to ensure data accuracy. After data entry, $10 \mathrm{CRFs}$ and data from the database of each trial were randomly 
selected and checked again to ensure consistency. After the final confirmation, all the data were imported into SPSS 13.0. After the logic programming check, the obviously incorrect data were modified. If the errors of data were from CRFs, the data manager then revised the data according to the answers from the researchers.

The SPSS 13.0 for Windows (SPSS Inc., Chicago, IL) was used for data analysis. Measurement data were indicated as mean $\pm \mathrm{SD}$. The acupuncture AEs related risk factors were analysed by unconditional logistic regression analysis. $P$ value was noted as significant when it was less than 0.05 .

\section{Results}

\section{General characteristics of patients}

A total of 1968 cases of patients (MI-RCT 475, FD-RCT 593, and BP-RCT 900) received acupuncture therapy in the three RCTs. The average age was $38.7 \pm 14.0$ years. The general characteristics of patient per trial were shown in Table 1.

\section{AEs and sequent treatments}

74 patients $(3.76 \%)$ experienced AE during the observational cycle. The general information of the patients with AEs was shown in Table 2. Overall, 74 cases of AEs were recorded. The most common adverse effects were needle-site bleeding (37.84\%) and subcutaneous bleeding (25.68\%) (Table 3). All types of AEs observed in this study have been reported previously $[7,10,15]$. It was worth mentioning that the following adverse events, lag needle, broken needle and forgotten needle, were not reported, and there were no SAEs relating to organ injury or nerve injury caused by malpractice.

59 patients $(79.73 \%)$ received subsequent treatment for recovery from AEs, including physiotherapy (19, $32.20 \%)$, self-treatment $(37,62.71 \%)$ and other effective methods (3, 5.08\%) (Table 3). 73 patients with AEs recovered in 2 weeks after subsequent treatment. One patient with dizziness did not fully recover within 2 weeks but reached full recovery one month after the cessation of acupuncture treatment. Of these 74 patients, three patients suffered either muscle spasm or dizziness or abdominal distension and withdrew from the study. The remaining 71 patients completed the entire treatment process. 64 of these 74 AEs cases were certainly due to the acupuncture treatment, and the remaining 10 cases were probably related to the acupuncture treatment.

\section{Logistic regression analysis of $\mathrm{AE}$ risk factors related with acupuncture treatment}

We analysed various potential risk factors associated with acupuncture treatment using logistic regression. We set the occurrence of AEs associated with acupuncture as a dependent variable (two categories, "1" for no $\mathrm{AE}$, "2" for AE), and age (continuous variable), sex (two categories, " 1 " for male, " 2 " for female ), education (multi-categorical variables, " 1 " for primary school education level, "2" for high school education level, "3" for university education level and above ), history of acupuncture treatment (two categories, "1" for without acupuncture experience, "2" for with acupuncture experience) and body mass index (continuous variable)

Table 1 General Characteristics and Distribution of Patients

\begin{tabular}{|c|c|c|c|c|c|}
\hline \multirow[t]{2}{*}{ Characteristic } & & \multicolumn{3}{|c|}{ Clinical Trials } & \multirow[t]{2}{*}{ Total(\%) } \\
\hline & & MI-RCT & FD-RCT & BP-RCT & \\
\hline \multirow[t]{2}{*}{ Gender } & Male & 82 & 173 & 474 & $729(37.0)$ \\
\hline & Female & 393 & 420 & 426 & $1239(63.0)$ \\
\hline \multirow[t]{2}{*}{ Marriage } & Unmarried & 134 & 191 & 211 & $536(27.2)$ \\
\hline & Married & 341 & 402 & 689 & $1432(72.8)$ \\
\hline \multirow[t]{3}{*}{ Educational Background } & Primary school & 34 & 39 & 90 & $163(8.3)$ \\
\hline & Middle school & 206 & 213 & 405 & $824(41.9)$ \\
\hline & University and above & 235 & 341 & 405 & 981 (49.9) \\
\hline \multirow[t]{2}{*}{ Acupuncture experience } & Not experienced & 346 & 588 & 491 & $1425(72.4)$ \\
\hline & Have experienced & 129 & 5 & 409 & $543(27.6)$ \\
\hline \multirow[t]{3}{*}{$B M I\left(k g / m^{2}\right)$} & $<18.5$ & 53 & 92 & 24 & $169(8.6)$ \\
\hline & $18.5 \sim 23.9$ & 342 & 428 & 495 & $1265(64.3)$ \\
\hline & $24.0 \sim 27.9$ & 80 & 73 & 381 & $534(27.1)$ \\
\hline \multirow[t]{5}{*}{ Age (year) } & $18 \sim 29$ & 178 & 215 & 258 & $651(33.1)$ \\
\hline & $30 \sim 39$ & 104 & 119 & 191 & $414(21.0)$ \\
\hline & $40 \sim 49$ & 90 & 118 & 175 & $383(19.5)$ \\
\hline & $50 \sim 59$ & 83 & 105 & 179 & 367 (18.7) \\
\hline & $60 \sim 69$ & 20 & 36 & 97 & $153(7.8)$ \\
\hline
\end{tabular}


Table 2 General characteristics of patients with AEs associated with acupuncture

\begin{tabular}{|c|c|c|c|c|c|}
\hline \multirow[t]{2}{*}{ Characteristics } & & \multicolumn{3}{|c|}{ Clinical Trials } & \multirow[t]{2}{*}{ Total (\%) } \\
\hline & & $\overline{\text { MI-RCT }}$ & FD-RCT & BP-RCT & \\
\hline \multirow[t]{2}{*}{ Gender } & Male & 6 & 1 & 12 & 19 (25.68) \\
\hline & Female & 29 & 10 & 15 & $54(72.97)$ \\
\hline \multirow[t]{2}{*}{ Marriage } & Unmarried & 6 & 1 & 2 & $9(12.2)$ \\
\hline & Married & 30 & 10 & 25 & $65(87.84)$ \\
\hline \multirow[t]{3}{*}{ Educational Background } & Primary school & 6 & 2 & 2 & $10(13.51)$ \\
\hline & Middle school & 12 & 3 & 14 & 29 (39.19) \\
\hline & University and above & 18 & 6 & 11 & $35(47.3)$ \\
\hline \multirow[t]{2}{*}{ Acupuncture experience } & Not experienced & 25 & 11 & 18 & $54(72.97)$ \\
\hline & Have experienced & 11 & 0 & 9 & $20(27.03)$ \\
\hline \multirow[t]{3}{*}{$B M I\left(\mathrm{~kg} / \mathrm{m}^{2}\right)$} & $<18.5$ & 3 & 1 & 0 & $4(5.4)$ \\
\hline & $18.5 \sim 23.9$ & 30 & 9 & 18 & $57(77.03)$ \\
\hline & $24.0 \sim 27.9$ & 3 & 1 & 9 & $13(17.57)$ \\
\hline \multirow[t]{5}{*}{ Age (year) } & $18 \sim 29$ & 8 & 1 & 4 & $13(17.57)$ \\
\hline & $30 \sim 39$ & 7 & 2 & 9 & $18(24.32)$ \\
\hline & $40 \sim 49$ & 6 & 2 & 8 & $16(21.62)$ \\
\hline & $50 \sim 59$ & 8 & 4 & 4 & $16(21.62)$ \\
\hline & $60 \sim 69$ & 7 & 2 & 2 & $11(14.86)$ \\
\hline
\end{tabular}

were set as covariates. All the variables were adopted into the model for analysis by using forward stepwise selection of independent variables for Wald Probability and Statistics Act for Logistic Regression analysis. Logistic regression equation was: Logit $(\mathrm{AE})=-3.782+0.018$ * age $+(-0.539$ * gender $)$; regarding the relative risk factor (Exp (B)) value terms, with one year increase, the possibility of AEs would increase 1.018-1 $=0.018$ times. From the perspective of gender, the possibility of AEs for females is $0.583-1=-0.417$ times that for males, i.e. the possibility of AEs for females is 0.417 times less than for males (Tables 4 and 5).

\section{Discussion}

With the increasing acceptance of acupuncture in more and more countries, governments and professional institutions, the safety of acupuncture is becoming of key concern in public discussion. Every acupuncture practitioner should objectively and factually report acupuncture AEs. According to the reports from United States, Germany, Britain, Korea, Japan et al [10,16-18], the incidence of AEs ranged from $0.671 \%$ to $11.4 \%$, with the most common acupuncture AEs being pain, fatigue, bleeding and haematoma. In this study, in order to ensure real and objective safety evaluation of acupuncture, we used a synchronous mode of observational studies to obtain the first-hand research data. The 1968 patients and their physicians were required to complete an acupuncture AE questionnaire and acupuncture adverse reports respectively. They were supervised and spot-checks were made during the whole process to minimize omission and selection bias. Since after-effects of acupuncture are known to exist, the study period to evaluate AEs included the acupuncture treatment process and the three months after treatment.

According to the type and frequency of AEs in acupuncture treatment established by Witt [19], ecchymoma and haemorrhage in needling position were the most common AEs, with an occurrence rate of more than $10 \%$. Our results were consistent with the Witt's. Previous literatures indicated there were 9 kinds of AEs, but the incidence and types of AEs found in our study were lower than former records. Comparing relevant literatures, we inferred that acupuncture AEs were associated with following factors.

Firstly, acupuncture AEs were related to the patient's own attributes. In this study, according to the results of logistic regression analysis, the occurrence of acupuncture AEs did not have significant correlation to BMI, educational background and acupuncture treatment experience of patients, but was correlated to age and sex. The older the patient, the higher was the risk of $\mathrm{AE}$, and male patients had slightly higher risk of $\mathrm{AE}$ than female patients. The analysis of risk factors for acupuncture AEs has only rarely been studied in previous literatures, usually only case reports have been reported. We have done these preliminary studies but still need to collect more demographic information and details to further this investigation.

Secondly, the types of acupuncture AEs were connected to the location and anatomical structure of the acupoints. In our study, the most common AEs of acupuncture were ecchymoma and bleeding in the needling position. From the occurrence percentage of the two 
Table 3 List of AEs in acupuncture and subsequent treatments

\begin{tabular}{|c|c|c|c|c|c|c|c|c|c|c|c|c|c|c|c|c|}
\hline \multicolumn{2}{|c|}{ Type of AEs } & \multicolumn{3}{|c|}{$\begin{array}{l}\text { Total patients with AEs } \\
(\mathrm{N}=74)\end{array}$} & \multicolumn{3}{|c|}{$\begin{array}{l}\text { Physiotherapy } \\
(\mathrm{N}=19)\end{array}$} & \multicolumn{3}{|c|}{$\begin{array}{l}\text { Self-treatment } \\
(\mathrm{N}=37)\end{array}$} & \multicolumn{3}{|l|}{$\begin{array}{l}\text { Others } \\
(\mathrm{N}=3)\end{array}$} & \multicolumn{3}{|c|}{$\begin{array}{l}\text { No treatment } \\
(\mathrm{N}=15)\end{array}$} \\
\hline & & $\begin{array}{l}\text { MI-RCT } \\
\text { n (\%) }\end{array}$ & $\begin{array}{l}\text { FD-RCT } \\
\text { n (\%) }\end{array}$ & $\begin{array}{l}\text { BP-RCT } \\
\mathrm{n}(\%)\end{array}$ & $\begin{array}{l}\text { MI-RCT } \\
\text { n (\%) }\end{array}$ & $\begin{array}{l}\text { FD-RCT } \\
\text { n (\%) }\end{array}$ & $\begin{array}{l}\text { BP-RCT } \\
n(\%)\end{array}$ & $\begin{array}{l}\text { MI-RCT } \\
\text { n (\%) }\end{array}$ & $\begin{array}{l}\text { FD-RCT } \\
\text { n (\%) }\end{array}$ & $\begin{array}{l}\text { BP-RCT } \\
\mathrm{n}(\%)\end{array}$ & $\begin{array}{l}\text { MI-RCT } \\
\text { n (\%) }\end{array}$ & $\begin{array}{l}\text { FD-RCT } \\
\text { n (\%) }\end{array}$ & $\begin{array}{l}\text { BP-RCT } \\
\mathrm{n}(\%)\end{array}$ & $\begin{array}{l}\text { MI-RCT } \\
\text { n (\%) }\end{array}$ & $\begin{array}{l}\text { FD-RCT } \\
\text { n (\%) }\end{array}$ & $\begin{array}{l}\text { BP-RCT } \\
\mathrm{n}(\%)\end{array}$ \\
\hline \multirow[t]{4}{*}{$\begin{array}{l}\text { Local } \\
\text { reactions }\end{array}$} & $\begin{array}{l}\text { Subcutaneous } \\
\text { haematoma }\end{array}$ & $6(8.11)$ & $3(4.05)$ & $\begin{array}{l}23 \\
(31.08)\end{array}$ & $6(31.58)$ & $3(15.79)$ & $3(15.79)$ & 0 & 0 & $9(24.32)$ & 0 & 0 & 0 & 0 & 0 & $\begin{array}{l}11 \\
(73.33)\end{array}$ \\
\hline & $\begin{array}{l}\text { Minor haemorrhage in } \\
\text { needling position }\end{array}$ & $\begin{array}{l}23 \\
(31.08)\end{array}$ & $2(2.70)$ & $3(4.05)$ & 0 & 0 & 0 & $\begin{array}{l}23 \\
(62.16)\end{array}$ & $2(5.41)$ & $3(8.11)$ & 0 & 0 & 0 & 0 & 0 & 0 \\
\hline & Subcutaneous bruise & $6(8.11)$ & $1(1.35)$ & 0 & $2(10.53)$ & $1(5.26)$ & 0 & 0 & 0 & 0 & 0 & 0 & 0 & $4(30.77)$ & 0 & 0 \\
\hline & $\begin{array}{l}\text { Prolonged pain at the } \\
\text { site of needling }\end{array}$ & 0 & $1(1.35)$ & 0 & 0 & $1(5.26)$ & 0 & 0 & 0 & 0 & 0 & 0 & 0 & 0 & 0 & 0 \\
\hline \multirow{5}{*}{$\begin{array}{l}\text { Systemic } \\
\text { reactions }\end{array}$} & Acupuncture fainting & 0 & $1(1.35)$ & $1(1.35)$ & 0 & 0 & 0 & 0 & 0 & 0 & 0 & $1(33.33)$ & 1 (33.33) & 0 & 0 & 0 \\
\hline & Abdominal distension & 0 & $1(1.35)$ & 0 & 0 & $1(5.26)$ & 0 & 0 & 0 & 0 & 0 & 0 & 0 & 0 & 0 & 0 \\
\hline & Dizziness/vertigo & 0 & $1(1.35)$ & 0 & 0 & 0 & 0 & 0 & 0 & 0 & 0 & $1(33.33)$ & 0 & 0 & 0 & 0 \\
\hline & Leg weakness & $1(1.35)$ & 0 & 0 & $1(5.26)$ & 0 & 0 & 0 & 0 & 0 & 0 & 0 & 0 & 0 & 0 & 0 \\
\hline & Muscle spasm & 0 & $1(1.35)$ & 0 & 0 & $1(5.26)$ & 0 & 0 & 0 & 0 & 0 & 0 & 0 & 0 & 0 & 0 \\
\hline
\end{tabular}


Table 4 Variables not included in the analysis of the model

\begin{tabular}{|c|c|c|c|c|c|}
\hline & & & Score & df & $p$ \\
\hline \multirow[t]{7}{*}{ Step 1} & \multirow[t]{6}{*}{ Variables } & Gender (1) & 4.055 & 1 & 0.044 \\
\hline & & Educational Background & 1.141 & 2 & 0.565 \\
\hline & & Educational Background (1) & 0.932 & 1 & 0.334 \\
\hline & & Educational Background (2) & 0.644 & 1 & 0.422 \\
\hline & & Acupuncture experience (1) & 0.966 & 1 & 0.326 \\
\hline & & $\mathrm{BMl}$ & 2.649 & 1 & 0.104 \\
\hline & \multicolumn{2}{|c|}{ Overall Statistics } & 7.407 & 5 & 0.192 \\
\hline \multirow[t]{6}{*}{ Step 2} & \multirow[t]{5}{*}{ Variables } & Educational Background & 0.824 & 2 & 0.662 \\
\hline & & Educational Background (1) & 0.554 & 1 & 0.456 \\
\hline & & Educational Background (2) & 0.607 & 1 & 0.436 \\
\hline & & Acupuncture experience (1) & 1.199 & 1 & 0.274 \\
\hline & & $\mathrm{BMI}$ & 1.422 & 1 & 0.233 \\
\hline & \multicolumn{2}{|c|}{ Overall Statistics } & 3.382 & 4 & 0.496 \\
\hline
\end{tabular}

types of AEs, subcutaneous haematoma accounted for 23/32 (71.88\%) in acupuncture treatment for Bell's palsy, and bleeding in the needling position accounted for 23/ $28(82.14 \%)$ in the therapy of migraine. The treatment plan of peripheral facial paralysis involved Yangbai (GB14), Dicang (ST4), Jiache (ST6), Xiaguan (ST7), Taiyang (EX-HN5), Quanliao (SI18) and other facial acupoints. As facial skin is thin and rich in blood vessels and superficial fascia are composed of loose connective tissue with plentiful vessels, it is easy to cause vessel damage or latent subcutaneous bleeding during needle manipulation. In the treatment of migraine, we chose Fengchi (GB20), Luxi (TE19), and Touwei (ST8) which are located on cranial region of head. As the cranial skin and superficial fascia have abundant blood circulation, and fibrous connective tissue under the superficial fascia and the vessel wall are closely linked, scalp bleeding is common after acupuncture and it is difficult to self-terminate as pressure needs to be applied to stop the bleeding. Thus, mastering the anatomical characteristics of acupoints accurately could not only prevent the occurrence of AEs but also contribute to treating adverse reactions actively and minimize the harm to patients.

Thirdly, AEs were related to the medical environment. The 1968 patients of three RCTs were from 16 hospitals in five provinces. These medical institutions are all public medical institutions and are top hospitals in China. All patients received acupuncture treatment using disposable needles in order to avoid cross-infection such as needle site infection, the emergence of sepsis and hepatitis, which can be caused by defective needle disinfection [20].

Fourthly, we inferred that the incidence of acupuncture AEs and acupuncture practitioner's experience and skills are probably and inversely related. In public hospitals of China, acupuncture physician should have obtained qualifications for a doctor's license before treating patients. This means that an acupuncture physician has received at least 4 years university education and at least 1 year clinical experience, not including the period of internship. The education background and working experience of all acupuncture practitioners in these three RCTs were very similar, they had undergone at least 8 years of acupuncture training and were qualified TCM doctors. Additionally, before the beginning of these clinical trials, all the acupuncture practitioners had received SOPs training courses of acupuncture operation plan. Only the acupuncturists who finished the training course and passed the qualification examination were able to take part in the clinical trial. During the process of acupuncture treatment, every trial unit was required to have at least two acupuncturists with more than 10 years of clinical experience, as technical advisors to provide guidance to young practitioners. We made the above inference by comparing the education background and qualifications of acupuncture physician reported in most of the foreign countries [5,21,22] (e.g. Modlock et al. described that midwives were trained in acupuncture according to the guidelines and performed acupuncture treatments five to six times a week) with those in our study. The level of training may be one of the reasons for the difference in frequency and types of acupuncture AEs between China and foreign countries.

Lastly, we considered that the mutual trust between doctors and patients could reduce the occurrence of AEs. The level of communication between doctors and patients during the process of clinical trials could be important so that all the patients were able to follow the

Table 5 Logistic Regression analysis for variables included in the Model

\begin{tabular}{|c|c|c|c|c|c|c|c|c|c|}
\hline & & \multirow[t]{2}{*}{ B } & \multirow[t]{2}{*}{ S.E. } & \multirow[t]{2}{*}{ Wald } & \multirow[t]{2}{*}{ df } & \multirow[t]{2}{*}{$P$} & \multirow[t]{2}{*}{$\operatorname{Exp}(B)$} & \multicolumn{2}{|c|}{ 95\% C.I. for EXP(B) } \\
\hline & & & & & & & & Lower & UppeI \\
\hline \multirow[t]{2}{*}{ Step $1^{a}$} & Age & 0.018 & 0.008 & 4.897 & 1 & 0.027 & 1.018 & 1.002 & 1.034 \\
\hline & Constant & -3.965 & 0.361 & 120.618 & 1 & 0.000 & 0.019 & & \\
\hline \multirow[t]{3}{*}{ Step $2^{\mathrm{b}}$} & Age & 0.018 & 0.008 & 4.719 & 1 & 0.030 & 1.018 & 1.002 & 1.034 \\
\hline & Gender(1) & -0.539 & 0.271 & 3.967 & 1 & 0.046 & 0.583 & 0.343 & 0.991 \\
\hline & Constant & -3.782 & 0.369 & 105.284 & 1 & 0.000 & 0.023 & & \\
\hline
\end{tabular}

Notes: a. Variable(s) entered on step 1: age; b. Variable(s) entered on step 2: gender. 
doctor's instruction, for example, do not receive acupuncture treatment in a fasting state or satiated state. To some degree, such measures could help reduce acupuncture syncope and aggravation of the disease caused by improper care.

The limitation of this study was it involved only migraine, functional dyspepsia, and Bell's palsy, without relating to other appropriate disease in acupuncture, and two-thirds data about acupuncture safety in this manuscript were from the trials for efficacy study.

\section{Conclusions}

Our study confirmed that acupuncture is a safe therapy. The risk factors for AEs were related to the patient's gender and age and the local anatomical structure at the acupoints. AEs could be reduced and mitigated by improving the medical environment, promoting the technical level of the acupuncture practitioners, and establishing a good relationship of mutual trust between doctor and patient. As acupuncture practitioners, we should be alert to the occurrence of acupuncture AEs. Once AEs taking place, we ought to handle and report them with scientific, rigorous and serious attitudes. Acupuncture training institutions, educators and medical decision-making departments should attach great importance to the provision of professional standards and skill training for acupuncture practitioners in order to minimize the risk of AEs.

\section{Additional material}

Additional file 1: Adverse Events Questionnaire for Patients.

Additional file 2: Adverse Events Reports for Acupuncturist

Additional file 3: Details of acupoints selected and manipulation in each trial.

\section{List of abbreviations}

RCT: Randomized controlled trial; AE: Adverse event; TCM: Traditional Chinese Medicine; MI-RCT: An RCT to treat migraine with acupuncture; FD: Functional dyspepsia; FD-RCT: An RCT to treat functional dyspepsia with acupuncture; BP-RCT: An RCT of acupuncture and moxibustion to treat Bell's palsy according to different stages; GCP: Good Clinical Practice; SAE, Serious adverse effect; SFDA: the State Food and Drug Administration; CRF: Case report form; SOP: Standard operating procedures; RCDMS: Remote Clinical Data Management Systems.

\section{Acknowledgements}

This research was supported by the National Basic Research Program of China (973 Program) (No.2006CB504501) and the "11th Five-Year Plan" National Key Technology R \& D program of China (No.2006BAl12B03). We gratefully acknowledge the National Clinical Trial Centre of Chinese Medicine (Chengdu GCP Centre) in China, for their support in monitoring and data management. We appreciate the cooperation for all of the following clinical centres: Affiliated Hospital of Chengdu University of Traditional Chinese Medicine, Affiliated Hospital of Hunan University of Traditional Chinese Medicine, Hubei University of Traditional Chinese Medicine, First Affiliated Hospital of Tianjin University of Traditional Chinese Medicine, and Qingdao Haici Medical Group.

\section{Author details}

${ }^{1}$ College of Acupuncture and Massage, Chengdu University of TCM,

Chengdu, Sichuan 610075, China. ${ }^{2}$ College of Clinical Medicine, Chengdu University of TCM, Chengdu, Sichuan 610075, China. ${ }^{3}$ College of Foreign Languages, Chengdu University of TCM, Chengdu, Sichuan 610075, China.

\section{Authors' contributions}

LZ drafted the manuscript. FWZ performed the data analysis. All authors contributed to the further writing of the manuscript as well as read and approved the final manuscript.

\section{Competing interests}

The authors declare that they have no competing interests.

Received: 10 September 2010 Accepted: 24 March 2011

Published: 24 March 2011

\section{References}

1. NIH Consensus Conference: Acupuncture. Jama 1998, 280:1518-1524

2. Brinkhaus B, Witt CM, Jena S, Linde K, Streng A, Wagenpfeil S, Irnich D, Walther HU, Melchart D, Willich SN: Acupuncture in patients with chronic low back pain: a randomized controlled trial. Arch Intern Med 2006, 166:450-457.

3. Ezzo J, Streitberger K, Schneider A: Cochrane systematic reviews examine P6 acupuncture-point stimulation for nausea and vomiting. J Altern Complement Med 2006, 12:489-495.

4. Iorno V, Burani R, Bianchini B, Minelli E, Martinelli F, Ciatto S: Acupuncture Treatment of Dysmenorrhea Resistant to Conventional Medical Treatment. Evid Based Complement Alternat Med 2008, 5:227-230.

5. Linde K, Streng A, Jurgens S, Hoppe A, Brinkhaus B, Witt C, Wagenpfeil S, Pfaffenrath V, Hammes MG, Weidenhammer W, et al: Acupuncture for patients with migraine: a randomized controlled trial. Jama 2005, 293:2118-2125.

6. Chung A, Bui L, Mills E: Adverse effects of acupuncture. Which are clinically significant? Can Fam Physician 2003, 49:985-989.

7. White A, Hayhoe S, Hart A, Ernst E: Adverse events following acupuncture: prospective survey of 32000 consultations with doctors and physiotherapists. Bmj 2001, 323:485-486.

8. Macpherson H, Scullion A, Thomas KJ, Walters S: Patient reports of adverse events associated with acupuncture treatment: a prospective national survey. Qual Saf Health Care 2004, 13:349-355.

9. Melchart D, Weidenhammer W, Streng A, Reitmayr S, Hoppe A, Ernst E, Linde K: Prospective investigation of adverse effects of acupuncture in 97733 patients. Arch Intern Med 2004, 164:104-105.

10. Yamashita $H$, Tsukayama $H$, Safety of acupuncture practice in Japan: patient reactions, therapist negligence and error reduction strategies. Evid Based Complement Alternat Med 2008, 5:391-398.

11. Chen X, Li Y, Zheng H, Hu K, Zhang H, Zhao L, Liu L, Mang L, Yu S: A randomized controlled trial of acupuncture and moxibustion to treat Bell's palsy according to different stages: design and protocol. Contemp Clin Trials 2009, 30:347-353.

12. Li Y, Liang F, Yu S, Liu X, Tang Y, Yang X, Tian X, Yan J, Sun G, Chang X, et al: Randomized controlled trial to treat migraine with acupuncture: design and protocol. Trials 2008, 9:57.

13. Zheng $H$, Tian XP, Li Y, Liang FR, Yu SG, Liu XG, Tang $Y$, Yang XG, Yan J, Sun GJ, et al: Acupuncture as a treatment for functional dyspepsia: design and methods of a randomized controlled trial. Trials 2009, 10:75

14. Zhao L, Liang FR, Li Y, Zhang FW, Zheng H, Wu X: Improved quality monitoring of multi-center acupuncture clinical trials in China. Trials 2009, 10:123.

15. Park SU, Ko CN, Bae HS, Jung WS, Moon SK, Cho KH, Kim YS, Park JM: Short-term reactions to acupuncture treatment and adverse events following acupuncture: a cross-sectional survey of patient reports in Korea. J Altern Complement Med 2009, 15:1275-1283.

16. Ernst $G$, Strzyz $H$, Hagmeister $H$ : Incidence of adverse effects during acupuncture therapy-a multicentre survey. Complement Ther Med 2003, 11:93-97.

17. Lao L, Hamilton GR, Fu J, Berman BM: Is acupuncture safe? A systematic review of case reports. Altern Ther Health Med 2003, 9:72-83. 
18. MacPherson $\mathrm{H}$, Thomas $\mathrm{K}$, Walters $\mathrm{S}$, Fitter $\mathrm{M}$ : A prospective survey of adverse events and treatment reactions following 34,000 consultations with professional acupuncturists. Acupunct Med 2001, 19:93-102.

19. Witt CM, Pach D, Brinkhaus B, Wruck K, Tag B, Mank S, Willich SN: Safety of acupuncture: results of a prospective observational study with 229,230 patients and introduction of a medical information and consent form. Forsch Komplementmed 2009, 16:91-97.

20. Ernst $E$, Sherman KJ: Is acupuncture a risk factor for hepatitis? Systematic review of epidemiological studies. J Gastroenterol Hepatol 2003, 18:1231-1236.

21. Uldbjerg N, Modlock J, Nielsen B: Acupuncture for the induction of labour: a double-blind randomised controlled study. BJOG 118:377.

22. Sherman KJ, Coeytaux RR: Acupuncture for Improving Chronic Back Pain, Osteoarthritis and Headache. J Clin Outcomes Manag 2009, 16:224-230.

doi:10.1186/1745-6215-12-87

Cite this article as: Zhao et al.: Adverse events associated with

acupuncture: three multicentre randomized controlled trials of 1968 cases in China. Trials 2011 12:87.

\section{Submit your next manuscript to BioMed Central} and take full advantage of:

- Convenient online submission

- Thorough peer review

- No space constraints or color figure charges

- Immediate publication on acceptance

- Inclusion in PubMed, CAS, Scopus and Google Scholar

- Research which is freely available for redistribution

Submit your manuscript at www.biomedcentral.com/submit
C Biomed Central 